TITLE:

\title{
NONLINEAR ACOUSTICS OF SUPERFLUID HELIUM
}

$\operatorname{AUTHOR}(S)$ :

Nemirovskii, S.K.

CITATION:

Nemirovskii, S.K.. NONLINEAR ACOUSTICS OF SUPERFLUID HELIUM. 数 理解析研究所講究録 1996, 949: 51-60

ISSUE DATE:

1996-05

URL:

http://hdl.handle.net/2433/60304

RIGHT: 


\title{
NONLINEAR ACOUSTICS OF SUPERFLUID HELIUM
}

\author{
S.K. Nemirovskii \\ Institute of Thermophysics, Prosp. Lavrentyeva, 1, \\ 630090, Novosibirsk, Russia \\ e-mail: nemirov@otani.thermo.nsk.su
}

\begin{abstract}
The results of theoretical investigation of first and second sound in HeII are reviewed. The variety of "standard" nonlinear phenomena are described such as nonlinear transformation of wave modes into one another, formation of shock fronts, nonlinear renormalization of sound velocity, stability and parametric transformation of nonlinear waves etc. The effects of damping and dispersion are studied. The possibility of self-focusing of the second sound in cubically nonlinear case as well as in the quadratically one is discussed. We also presented the investigation of stochastic wave fields and acoustic turbulence. In conclusion some of the open problems and the paths for further development touched upon are discussed.
\end{abstract}

\section{Equations of hydrodynamics of superfluid Helium}

\subsection{Two-fluid hydrodynamics.}

In this section the hydrodynamics of superfluid Helium was introduced and described. From point of view of hydrodynamics HeII can be imagined as a mix of two liquids (see e.g. ref. [1]). One of them is superfluid component which behaves as an ideal fluid with zero viscosity as well as with zero entropy. Another, normal component behaves as usual viscous fluid. Hydrodynamics of such system can be formulated in terms of density $\rho$, entropy $S$, superfluid velocity $\mathrm{v}_{s}$ and quantity $\mathrm{j}_{0}$ which is the momentum density in the reference system connected with the superfluid component motion. The energy density in this system $E_{0}$ has a following form.

$$
E=\rho v_{s}^{2} / 2+\mathbf{v}_{s} \mathbf{j}_{0}+E_{0}\left(\rho, S, \mathbf{j}_{0}\right)
$$


The differential of the quantity $E_{0}\left(\rho, S, \mathbf{j}_{0}\right)$ is

$$
d E_{0}=T d S+\mu d \rho+\mathbf{v}_{n s} d \mathbf{j}_{0}
$$

The set of nondissipative equations of motion consists of the following four relations:

$$
\begin{aligned}
& \frac{\partial \rho}{\partial t}+\operatorname{div} \mathbf{j}=0 \\
& \frac{\partial j_{i}}{\partial t}+\frac{\partial \Pi_{i k}}{\partial x_{k}}=0 \\
& \frac{\partial S}{\partial t}+\operatorname{div} S \mathbf{v}_{n}=0 \\
& \frac{\partial \mathbf{v}_{s}}{\partial t}+\operatorname{grad}\left(\mu+\frac{\mathbf{v}_{s}^{2}}{2}\right)=0
\end{aligned}
$$

They represent the conservation low for density, for relative momenta and for entropy, where is taking into account that entropy is transferred only with normal component. The last equation for superfluid velocity $\mathbf{v}_{s}$ reflects the fact that driving force for superfluid component is a chemical potential $\mu\left(\rho, S, \mathbf{v}_{n}-\mathbf{v}_{s}\right)$

The full momenta $\mathbf{j}$ is

$$
\mathbf{j}=\rho_{s} \mathbf{v}_{s}+\rho_{n} \mathbf{v}_{n}
$$

The momentum flux density is defined as follows

$$
\Pi_{i k}=\rho_{n} \mathbf{v}_{n i} \mathbf{v}_{n k}+\rho_{s} \mathbf{v}_{s i} \mathbf{v}_{s k}+p \delta_{i k}
$$

where $p$ is the pressure.

The relations written above describe hydrodynamics of superfluid helium and accordingly can be regarded as a base for study of problems of linear and nonlinear acoustics.

\subsection{The Hamiltonian form of the equations of motion.}

An alternative to the Relations (3)-(6) is the representation of the equations of motion in so-called Hamiltonian form. The method of of the Hamiltonian formalism is very effective for studying nonlinear waves (see e.g. Refs [2] and [3]). For case of HeII the Hamiltonian formalism was elaborated in work [4]. There are two pairs of canonically conjugate variables $(\rho, \alpha),(S, \beta)$ (in potential flow) which are related to earlier introduced variables as follows.

$$
\mathbf{j}_{0}=S \operatorname{grad} \beta, \quad \mathbf{v}_{s}=\operatorname{grad} \alpha .
$$


In new variables the equation of motion of HeII acquire the canonical form

$$
\begin{array}{ll}
\dot{\rho}=\frac{\delta H}{\delta \alpha}, & \dot{\alpha}=-\frac{\delta H}{\delta \rho} ; \\
\dot{S}=\frac{\delta H}{\delta \beta}, & \dot{\beta}=-\frac{\delta H}{\delta S}
\end{array}
$$

where the Hamiltonian $H$ is the energy $E$ (1)expressed in canonical variables. Furthermore, it is possible to introduce other two other pairs, so called normal coordinates $a_{\mathbf{k}_{\mathbf{i}}}^{\nu_{j}}, \operatorname{mbox}($ $\nu= \pm 1,2)$ which are Fourier components of some linear combination of initial variables. Equation of motion have a following form:

$$
i \dot{a_{\mathbf{k}}^{\nu}}=\operatorname{sign} \frac{\delta H}{\delta a_{-\mathbf{k}}^{-\nu}} \text {. }
$$

The Hamiltonian $H$ is a series in integral powers of the variables $a_{\mathbf{k}_{\mathbf{i}}}^{\nu_{j}}$. It has the following form up to third order inclusively:

$$
H=\int \omega_{\mathbf{k}}^{1} a_{\mathbf{k}}^{1} a_{-\mathbf{k}}^{-1} d^{3} \mathbf{k}+\int \omega_{\mathbf{k}}^{2} a_{\mathbf{k}}^{2} a_{-\mathbf{k}}^{-2} d^{3} \mathbf{k}+\int V_{\mathbf{k}_{1} \mathbf{k}_{2} \mathbf{k}_{3}}^{\nu_{1} \nu_{2} 1 \nu_{3}} a_{\mathbf{k}_{1}}^{-\nu_{1}} a_{\mathbf{k}_{2}}^{-\nu_{2}} a_{\mathbf{k}_{3}}^{-\nu_{3}} d^{3} \mathbf{k}_{1} d^{3} \mathbf{k}_{2} d^{3} \mathbf{k}_{3} .
$$

It can be seen from relations (12)-(13) that normal coordinates in linear case corresponds to oscillation of medium (sounds ) and that there are two types of independent sound waves, so called first and second sounds. We describe their physical meaning later in next section. In nonlinear case these sounds interact due to presence of terms of third (and higher) order in Hamiltonian $H$.

\section{One-dimensional nonlinear waves}

\subsection{Reimann invariants.}

In this section we will study the laws of propagation of one-dimensional (along axis $x$ ) nonlinear sound waves of the first and second sounds. Let us introduce the following variables $\varphi_{1}=\rho^{\prime}$ - perturbation of density, $\varphi_{2}=v$ - mean-mass velocity, $\varphi_{3}=S^{\prime}$ - perturbation of entropy and $\varphi_{4}=w$ - relative velocity. Up to terms of second order in these quantities the set of equation can be written as follows:

$$
\frac{\partial \varphi_{i}}{\partial t}+\sum_{j} A_{i j}\left(\varphi_{i}\right) \frac{\partial \varphi_{j}}{\partial x}=0 . \quad(i, j=1,2,3,4)
$$

The dependence of the matrix elements $A_{i j}\left(\varphi_{i}\right)$ is no stronger than linear (see [5]).

In [5] there was shown that under some supposition for waves travelling in one direction it was possible to introduce Reimann invariants $I_{1}$ and $I_{3}$ which depend only on variables $\varphi_{1}=\rho^{\prime}$ and $\varphi_{3}=S^{\prime}$ 


$$
\begin{aligned}
& I_{1}=\rho^{\prime}+\alpha_{1} \rho^{\prime 2}+\alpha_{2}{\sigma^{\prime}}^{2} \\
& I_{3}=\sigma^{\prime}+\beta_{1}{\sigma^{\prime 2}}^{2}+\beta_{2} \rho^{\prime} \sigma^{\prime}
\end{aligned}
$$

and satisfy to the following equations:

$$
\begin{aligned}
& \frac{\partial I_{1}}{\partial t}+\xi^{1}\left(\varphi_{i}\right) \frac{\partial I_{1}}{\partial x}=0 \\
& \frac{\partial I_{3}}{\partial t}+\xi^{3}\left(\varphi_{i}\right) \frac{\partial I_{3}}{\partial x}=0 .
\end{aligned}
$$

Characteristics $\xi^{1}\left(\varphi_{i}\right)$ and $\xi^{3}\left(\varphi_{i}\right)$ correspond nonlinear velocities of the first and second sounds. It can be seen from Eqs. (17)-(18) that Reimann invariants corresponds to the waves travelling along the axis $\mathrm{x}$. In linear case the first invariant (first sound) describes to oscillations of density (and pressure) and mean-flow velocity and, therefore, represents usual sound. Conversely, the second Reimann invariants leads to perturbations of entropy (and temperature) and counterflow velocity. Existence of waves of temperature is the unique phenomenon specific for superfluid helium. It also can be seen from Rels. (15)-(18) that in linear case waves of pressure and temperature are independent whereas in nonlinear case there is a coupling between them. In particular heating the wall one will detect both waves, the main pulse of the temperature disturbance and some "precursor" which propagates with the much larger speed of first sound and which transfers perturbations of pressure. Details of this effects as well as exact quantitative results are described in papers [3], [5].

\subsection{Evolution of intense waves.}

Let us consider in more details nonlinear evolution of the second sound wave. In order to do it we express the second Reimann invariant via normal velocity $v_{n}$, and take into account dissipation as well as dispersion ( near $T_{\lambda}$ ). The last step can be performed using method of quasisimple waves (see e.g. [3], [6]). After according calculations we arrive at the following equation of evolution of the normal velocity

$$
\frac{\partial v_{n}}{\partial t}+\left[c_{2}+\alpha(T) v_{n}\right] \frac{\partial v_{n}}{\partial x}=\mu_{2} \frac{\partial^{2} v_{n}}{\partial x^{2}}+\mu^{3} \frac{\partial^{3} v_{n}}{\partial x^{3}}
$$

Relations of such kind is frequently encountered in the theory of nonlinear waves and is called Kortweig-de Vries - Burgers equation (KdVB).

Neglecting both nonlinear and dissipative terms one concludes that Rel. (??) describes usual wave of unchanged profile. However nonlinear effects lead to deformation of the wave profile. In particular term $\left.\alpha(T) v_{n}\right] \frac{\partial v_{n}}{\partial x}$ is responsible for steepening of shape of the wave pulse. Coefficient $\alpha(T)$ is some complicated function of the temperature, it can be both positive and negative and it is equal to zero at temperature about $1.885 \mathrm{~K}$.. Therefore when $\alpha(T)>0$ the shock front 
forms on the leading part of the pulse. In regions where $\alpha(T)<0$ the steepening and formation of shock front occurs on the back edge of the wave profile. This very interesting phenomenon was observed in various experiments. At the temperature $1.885 \mathrm{~K}$ where $\alpha(T)=0$ there is no influence of second order nonlinearity and evolution of wave is determined by next cubic nonlinearity.

\subsection{Cubically nonlinear effects.}

A cubically nonlinear medium has one remarkable property. It can support steady-state (e.g. ,the profile does not change) monochromatic wave. This problem was studied in work [???]. According this work let us introduce the complex envelope of the wave packet $\Psi$, which is defined via normal coordinates $a_{\mathbf{k}}^{ \pm 2}$

$$
\Psi(\mathbf{r}, t)=\frac{1}{(2 \pi)^{3} / 2} \int a_{\mathbf{k}}^{ \pm 2} \exp i\left(\mathbf{k}-\mathbf{k}_{0}\right) \mathbf{r} d^{3} \mathbf{k} .
$$

Equation for quantity. $\Psi$ can be derived from Hamiltonian equation (12)-(13). The Hamiltonian $H$ was found by simply going through all contribution to the energy in given approximation. Using $H$ obtained in this way we arrived at the following equation:

$$
i \frac{\partial \Psi}{\partial t}=\omega_{\mathrm{k}_{0}}^{2} \Psi+V|\Psi|^{2} \Psi .
$$

It is famous nonlinear Shredinger equation. It has the uniform obvious uniform solution as follows:

$$
\Psi(t)=\Psi_{0}(t) \exp i \Omega\left(\mathrm{k}_{\mathbf{0}}, \Psi_{0}\right) t
$$

which corresponds to monochromatic wave. The frequency of this wave depends however both on wave vector $\mathrm{k}$ and on the amplitude $\Psi_{0}$ ( the nonlinear dispersion law). Numerical analysis shows that nonlinear correction to the second velocity sound is equal to

$$
\delta c_{2}=-1.09 \frac{k_{0}}{\rho}|\Psi|^{2}
$$

Thus the nonlinear wave moves more slowly than linear wave. It is important to note that main contribution to this effect appeared due to interaction (Doppler shift) of initial second sound with first sound generated in the system (see section 2.1). 


\section{Multi-dimensional wave packets.}

We will describe now behavior of weakly non-one-dimensional packets of nonlinear waves of second sound. For waves with an infinitesimal amplitude taking the transverse nonuniformity into account results in diffraction phenomena. In the case of finite amplitudes a number of fundamentally new effects, connected with the combined action of nonlinear and diffraction terms in the equations of motion, can arise.

We begin with cubically nonlinear medium described in previous section. It was shown in work [7] that complex envelope nonlinear $\Psi(\mathbf{r}, t)$ of monochromatic waves, weakly modulated in transverse direction, evolve obeying to following equation (in the reference frame moving with second sound velocity $c_{2}$ )

$$
2 i k_{0} \frac{\partial \Psi}{\partial x}-\triangle_{\perp} \Psi=\omega_{\mathrm{k}_{0}}^{2} \Psi+\frac{2 k_{0}}{c_{2}} V|\Psi|^{2} \Psi
$$

Analyses of this equation (see[7]) shows that waves have a tendency self-focusing. The physical meaning of this effect is following. As it was shown in Sec 2.3 the term in r.h.s. is positive what means that the velocity of propagation of wave decreases with the amplitude. As a result at the periphery of the wave packet ( far from axis $x$ ) where the amplitude is smaller, the velocity of the wave is higher than on the axis of the beam. Thus the wavefront bends and focusing of the packet starts. As a result the amplitude on the axis increases, which results in an greater difference between the velocity of propagation of the peripheral and central parts. This intensifies the focusing effects even more. The diffraction term $\triangle_{\perp} \Psi$ in Eq. (24)results in spreading of the packet in the transverse direction. For this reason, to observe self-focusing the energy flux must exceed some threshold value $I_{c r}$, which is about $0.610^{7} \nu^{-2} w$, where $\nu$ is the frequency.

Thus we showed that nonlinear monochromatic waves of second sound in cubically nonlinear medium are undergoing to self-focusing effects, and main reason of that is that velocity of propagation of nonlinear wave is smaller than of a linear one.

Some analoguos effects can take place in quadratically nonlinear medium where $\alpha(T)$ is negative what corresponds to slowing of nonlinear wave. The evolution equation for normal velocity $v_{n}$ ( in the reference frame moving with second sound velocity $c_{2}$ ) can be derived from KdVB equation (19) (see Ref. [3] and [6]).

$$
\frac{\partial}{\partial x}\left(\frac{\partial v_{n}}{\partial t}+\alpha(T) v_{n} \frac{\partial v_{n}}{\partial x}-\mu_{2} \frac{\partial^{2} v_{n}}{\partial x^{2}}\right)=\frac{c_{2}}{2} \triangle_{\perp} v_{n} .
$$

Compared with the nonlinear parabolic equation (24) Eq. (25) has not been studied much. One reason for this is that there are no steady waves with constant profile ( $\operatorname{see} \mathrm{Sec} 2.2$ ). There are ,however, a number of numerical simulations of Eq. (25) (see e.g. book [6]), which pointed out to self-focusing effects, at least, before formation of shock front. However after forming shock front the situation is extremely involved and behavior of wave is not studied. 


\section{Stability of nonlinear waves.}

\subsection{Nonlinear transformation of first sound into second sound.}

The study of of stability of solutions is an important part of the theory of nonlinear waves. First of all, this permits determination the region of values of the parameters for which the solution found is realized. Second, the possible instability of the waves is related with the nonlinear character of the equations of motion.

There is one other aspect to the question of stability of the waves. Suppose that a wave of first sound is excited in helium. Suppose further that small disturbances, associated with the second-sound mode, are unstable in the presence of this this first sound. This effect can be interpreted as the generation of second sound by first sound (see [4]). Authors of [4] studied stability of monochromatic wave of first sound $a_{\mathrm{k}_{1}}^{1}$ with respect two following nonlinear processes, decomposition process and Cherenkov process.

$$
\begin{aligned}
& a_{\mathbf{k}_{1}}^{1} \Longrightarrow a_{\mathbf{k}_{2}}^{2}+ \\
& a_{\mathbf{k}_{3}}^{2} a_{\mathbf{k}_{1}}^{1} \Longrightarrow a_{\mathbf{k}_{2}}^{1}+a_{\mathbf{k}_{3}}^{2}
\end{aligned}
$$

It is well known from general results (see Ref. [2] ), that instability develops under conditions of resonance, which e.g. for decomposition processes have a following form:

$$
\begin{aligned}
& \omega_{\mathbf{k}_{1}}^{1}=\omega_{\mathbf{k}_{2}}^{2}+\omega_{\mathbf{k}_{3}}^{2} \\
& \mathbf{k}_{1}=\mathbf{k}_{2}+\mathbf{k}_{3} .
\end{aligned}
$$

Studying of these conditions allowed the authors to draw the following conclusion. Almost for all temperatures the Cherenkov process dominates, e.i.the initial wave should decay into first sound and second sound, which propagates in opposite (with respect to initial wave) direction. Authors also calculated the critical amplitudes of this process.

\subsection{Stability of a pressure shock waves.}

As it was already mentioned, in a quadratically nonlinear medium any wave forms a shock front. Therefore it is of great interest to study the condition of stability of shock front with respect to generation of the first and second sound as well as to arbitrary distortion of an initially plain shock front. This problem was studied in paper [8]. The problem was solved based on the Hamiltonian equations of motion in the class of generalized function. In an idealized shock wave - a step moving from left to right - the hydrodynamics, for instance mean-flow velocity $v$, variables are proportional to a unit step function 


$$
v \propto \theta(x-U t) .
$$

Furthermore it is known that unit step function has a following Fourier representation:

$$
\mathcal{F} \theta(x-U t) \propto \frac{\exp \left(-i k_{x} U t\right)}{k_{x}+i 0}
$$

Where the term $+i 0$ gives a rule for circumscribing the pole. Equations for small perturbation $b_{\mathbf{k}_{1}}$ of any of listed nature in the presence of shock wave can be written down in Fourier space as follows ( see Ref. [8]):

$$
\frac{\partial b_{\mathbf{k}_{1}}}{\partial t}+i\left(\omega_{\mathbf{k}_{1}}^{\nu}-k_{x} U\right) b_{\mathbf{k}}+\frac{1}{\pi i} \int \frac{V_{\mathbf{k}_{1}, \mathbf{k}_{2}}^{\nu} b_{\mathbf{k}_{1}} d^{3} \mathbf{k}_{1}}{k_{2 x}-k_{x}+i 0}=0 .
$$

Here vertices $V_{\mathbf{k}_{1}}^{\nu}$ depend on what concrete type of perturbations we study. They are rational functions of variables $\mathbf{k}_{1}, \mathrm{k}_{2}$. This circumstance allows to to reduce the solving of Eq. (32) to the reconstruction of a piecewise- analytical function (of the complex variable $k_{x}$. The calculations performed in Ref. [8] lead to the following results. Small perturbations of both first and second sound are neutral i.e. and corresponding solution just describes how they go through shock front. As for small distortion of shock front, they decay exponentially with decrement $\Delta v k_{\perp}$, where $\Delta v$ is amplitude of shock wave and $k_{\perp}$ is inverse size of "ripple" on the shock front surface.

\section{Stochastical nonlinear wave processes.}

The last section of the paper is devoted to stochastical wave- a set of wave with chaotic phaseswhich can appear in system due to various nonlinear processes of instabilities or due to random pumping of wave energy into the volume of the liquid.

suppose that we have some source of wave energy that generates harmonics with a characteristic wave number of the order of $k_{+}$, which is supposed to be (in order of magnitude) the inverse size of the system, $k_{+} \sim L^{-1}$. As a result of nonlinear processes harmonics with higher values of $k$, which, in their turn, generate still higher harmonics, appear in the system. For very large values of $k$, of the order of $k_{-}$, viscous terms come into play in the equations of motion, and waves with momenta $k>k_{-}$. decay rapidly. Ultimately some distribution of the wave, which is characterized by the transfer of energy from large- scale scale motion to small-scale motion, is established in $k$-space. This picture is typical for turbulent phenomena, and since we are talking about sound waves it is called acoustical turbulence.

Following paper [9], in order to describe quantitatively acoustical turbulence let us introduce the Fourier transforms of pair correlation function of normal coordinates $a_{\mathbf{k}}^{\nu}$ - so called spectra $n_{\mathbf{k}}^{\nu}$. They are defined as follows:

$$
\left\langle a_{\mathbf{k}_{1}}^{\nu} a_{\mathbf{k}_{2}}^{-\nu}\right\rangle=n_{\mathbf{k}}^{\nu} \delta\left(\mathbf{k}_{1}+\mathbf{k}_{2}\right) \text {. }
$$


As it was shown in [9], under supposition that nonlinearity is not too strong, the dynamics of spectra obeys to set of kinetic equations in $k$-space. Thus the problem is reduced to seeking for the solution of the kinetic equation which satisfies to constant flux of energy in $k$-space. If we assume that $k_{+}$is very small, whereas $k_{-}$very large, then in the range, which is far both from $k_{+}$and from $k_{-}$(so called inertial interval) there are no no characteristic dimension for $k$. Taking into account that the coupling constants $V_{\mathbf{k}_{1} \mathbf{k}_{2} \mathbf{k}_{3}}^{\nu_{1} \nu_{2} 1 \nu_{3}}$ (see Sec. 1.2) are homogeneous functions of its arguments $\mathbf{k}_{i}$ we are able to assume that solution should be scaling invariant.

$$
n_{\mathrm{k}}^{1}=A k^{s} \quad n_{\mathrm{k}}^{2}=B k^{s}
$$

Further analyses, which supposed some transformation of the kinetic equations leads to remarkable result that quantity $s$ depends only on fundamental properties of the system such as dimension of space, dispersion laws of first and second sounds and power of uniformity of coupling constants and equal to

$$
s=-9 / 2
$$

It is interesting to note that in spite of general nonequilibrium the first and second sound mode are in equilibrium, there is no flux of energy from the one mode to the another one. One more interesting result is that most part of energy is stored in the second "soft" wave mode. In paper [9] it was also shown that HeII, in which the fields of chaotic waves are developed, possesses unique acoustical properties. In particular there appears additional dissipation of the both second and first sound as well as dispersion of the sounds velocities.

\section{ACKNOWLEDGMENTS.}

This report had been prepared during visit of author to Japan. I would like to thank Japan Society for the Promotion of Science (JSPS) for the organization of this travel. I also would like to thank participants of scientific seminar of Prof. M. Murakami at Tsukuba University for useful discussion of many of the results represented in this paper. Partly some of worked exposed here were supported by Grants No. RPKOOO and No. RPK300 from International Science Foundation.

\section{REFERENCES}

1.Khalatnikov, I.M., 1965, An Introduction to the Theory of Superfluidity, (W.A. Benjamin, New York/Amsterdam).

2. Zakharov, V.E., 1974, Radiophys. Quantum Electron 17, 326.

3. Nemirovskii, S.K., 1990, Sov. Phys.-Usp. 33, 429.

4. Pokrovskii, V.L. and I.M. Khalatnikov, 1976, Sov. Phys.-JETP ,44, 1036.

5. Nemirovskii, S.K., 1984, Sov. Phys.-JETP, 59, 1215.

6. Rudenko, O.V., Soluyan, S.I.,1977, Theoretical Foundations of Nonlinear Acoustics, Consultant Bureau, New-York). 
7. Nemirovskii, S.K., 1978, Sov. J. Low Temp. Phys., 4, 577.

7. Nemirovskii, S.K., 1985, Sov. J. Low Temp. Phys., 11, 431.

8. Nemirovskii, S.K., 1986, Sov. Phys.-JETP, 63, 1186. 